\title{
Medidas de segregación socioespacial: discusión metodológica y aplicación empírica sobre ciudades medias argentinas
}

\author{
Santiago Linares* \\ Universidad Nacional del Centro de la Provincia de Buenos Aires, Tandil, Argentina
}

\section{RESUMEN}

En el período actual, caracterizado por una gran difusión de las aplicaciones informáticas y técnicas de análisis espacial insertas en las Tecnologías de la Información Geográfica, existen abundantes herramientas que permiten a cualquier interesado calcular una diversidad de índices de segregación socioespacial. No obstante, esto no resulta suficiente para garantizar una correcta implementación e interpretación de las medidas disponibles en las diferentes aplicaciones. Este artículo pretende contribuir en tres aspectos. En primera instancia, examinar los fundamentos teóricos y metodológicos sobre los cuales se han desarrollado las medidas de segregación socioespacial; en segunda, implementar dos aplicaciones informáticas desarrolladas específicamente para su medición; y, en tercera instancia, ejecutar los cálculos para 10 ciudades medias de la Provincia de Buenos Aires (Argentina). Los resultados permiten corroborar la extraordinaria capacidad de las nuevas Tecnologías de la Información Geográfica como base metodológica para los análisis de segregación socioespacial. También evidencian que las ciudades, a medida que aumentan su rango, exhiben un patrón urbano más segregado, de la misma manera que se detecta cómo la expresión cuantitativa de este fenómeno, aunque sea medida en base a las mismas circunstancias y fuentes de datos, varía según el índice seleccionado y dimensión específica de que se trate.

* Magíster en Teledetección y Sistemas de Información Geográfica, Universidad Nacional del Centro de la Provincia de Buenos Aires, Argentina; doctor en Geografía, Universidad Nacional del Sur, Argentina. Becario posdoctoral, Comisión Nacional de Investigaciones Científicas y Técnicas (Conicet) de Argentina. Docente del Departamento de Geografía, Facultad de Ciencias Humanas, Universidad Nacional del Centro de la Provincia de Buenos Aires. Correo electrónico: slinares@fch.unicen.edu.ar. 


\section{Palabras clave}

Medidas de segregación socioespacial, uniformidad y exposición, segregación y rango urbano, ciudades medias argentinas, Sistemas de Información Geográfica

\section{Socio-spatial segregation measures: Methodological discussion and empirical application on medium-sized Argentine cities}

\section{ABSTRACT}

In the current period, marked by a great many computer applications and spatial analysis techniques embedded in Geographic Information Technology, there are plenty of tools that allow any interested person to calculate a variety of socio-spatial segregation indices. Nevertheless, this is not enough to guarantee a correct implementation and interpretation of the measures available in the different applications. This article aims to contribute in three aspects. In the first place, examine the theoretical and methodological grounds on which the measures of socio-spatial segregation have developed; secondly, implement two IT applications developed specifically in order to measure them; and thirdly, make the calculations for 10 medium-sized cities in the province of Buenos Aires (Argentina). The results corroborate the extraordinary capacity of the new Geographic Information Technologies as a methodological basis for the analysis of socio-spatial segregation. They also demonstrate that cities, as they increase their rank, exhibit a more segregated urban pattern; likewise they detect how the quantitative expression of this phenomenon, although it is measured based on the same circumstances and data sources, varies according to the index selected and the specific dimension concerned.

\section{Keywords}

Socio-spatial segregation measures, uniformity and exposure, segregation and urban rank, medium-sized Argentine cities, Geographic Information Systems

\section{Introducción}

Las investigaciones sobre la segregación socioespacial avanzan sobre tres ejes analíticos: las investigaciones sobre los patrones de segregación y las metodologías implementadas para tal fin; las investigaciones sobre las causas de la segregación, y las investigaciones sobre las consecuencias de la segregación. En este artículo se presenta una revisión de las metodologías implementadas en las investigaciones 
sobre los patrones de segregación, destacando aquellos aportes que representan mejoras sustantivas en la medición e interpretación de este fenómeno.

Para enmarcar correctamente la evolución de las medidas de segregación es necesario definir previamente qué entendemos por segregación. A partir de los antecedentes bibliográficos (Burgess, 1925; Castells, 1972; White, 1983; Grafmeyer, 1994; Musterd y Ostendorf, 1998; Rodríguez, 2001; Schteingart, 2001; Sabatini, 2003; Sposito, 2005), podríamos afirmar que existe un concenso generalizado en cuanto a que la segregación hace referencia a la falta de uniformidad en la distribución de los grupos o categorías sociales a través del espacio urbano, y al agrupamiento de los sujetos según atributos específicos en aglomerados con tendencia a la homogeneización en su interior y a la reducción de las interacciones con el resto de los grupos. Si bien en la mayoría de los casos existe una fuerte correlación entre estas dimensiones ${ }^{1}$ objetivas de la segregación socioespacial, la presencia de un tipo de segregación no implica la presencia de la otra; es por ello que deben ser consideradas como dimensiones independientes.

Mientras que la uniformidad espacial se refiere al grado en que los grupos están igualmente distribuidos en el espacio residencial, la exposición espacial se refiere a la medida en que los miembros de un grupo se encuentran con miembros de otro grupo en un entorno residencial próximo. La exposición espacial depende, en gran parte, de la composición general de la población en la ciudad objeto de la investigación, mientras que la uniformidad espacial es independiente de la composición de la población.

Para ver que la uniformidad y la exposición son conceptualmente distintas, se presentan cuatro patrones residenciales hipotéticos en la Figura $\mathrm{N}^{\circ} 1$, en donde cada cuadrante representa un escenario urbano y cada punto representa un hogar habitado por miembros del grupo blanco o negro según corresponda. En la mitad superior del gráfico hay dos patrones en los cuales los hogares del grupo blanco y negro se distribuyen uniformemente por todo el espacio. Ambos modelos tienen niveles altos de uniformidad espacial (o bajos niveles de aglomeración espacial). En el patrón de la parte superior derecha, sin embargo, existen más hogares del grupo negro en el entorno próximo de cada hogar del grupo blanco (y viceversa)

\footnotetext{
Un trabajo de sistematización y discusión teórica y metodológica notable para delimitar las dimensiones objetivas de la segregación, fue el realizado por Massey y Dentons (1988) quienes, mediante un análisis de factores, definieron que el problema del reparto de un grupo de población en una ciudad puede abordarse a partir de cinco dimensiones: uniformidad, exposición, aglomeración, concentración y centralización. No obstante, un buen número de autores van a explicar la necesidad de prescindir de las últimas tres, tanto por inconsistencias teóricas y metodológicas en sí mismas (Reardon y O'Sullivan, 2004) como por incompatibilidades con respecto al contexto urbano latinoamericano (Rodríguez, 2005; Sabatini y Sierralta, 2006).
} 
que en el patrón de la parte superior izquierda. Esto significa que la exposición blanco-negro es superior en la derecha, y el aislamiento blanco es superior en la izquierda. Por su parte, en la mitad inferior del gráfico ambos patrones muestran una mayor aglomeración: el cuadrante inferior derecho muestra una idéntica exposición blanco-negro al mencionado anteriormente, pero se observan cambios significativos al seleccionar casos específicos sobre el cuadrante inferior izquierdo.

Figura $\mathrm{N}^{\circ}$ 1. Dimensiones objetivas de la segregación socioespacial

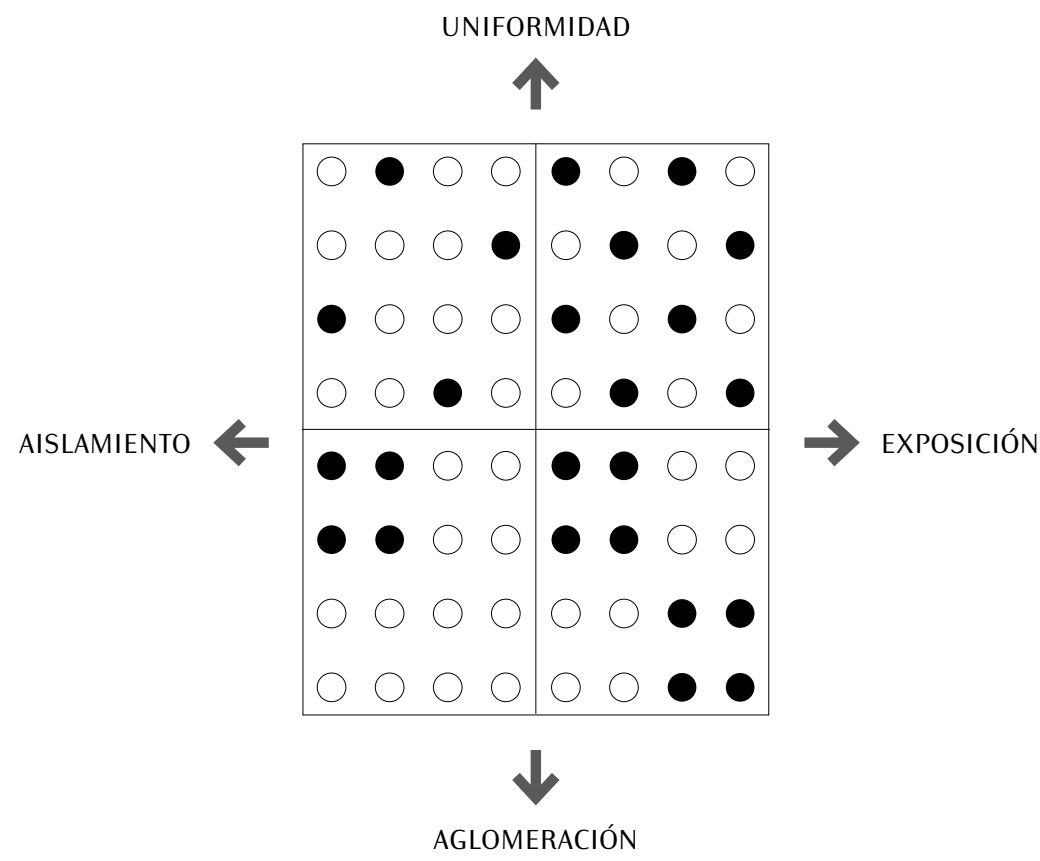

Fuente: elaboración propia; adaptado de Reardon y O’Sullivan (2004).

\section{Críticas a los índices clásicos basados en unidades censales}

Si bien los primeros antecedentes metodológicos para analizar los patrones espaciales de segregación (concentración y desigualdad) se basan en los procedimientos genéricos surgidos desde la econometría, como la curva de Lorenz y el coeficiente de concentración de Gini, es posible afirmar que los métodos más usuales a lo largo de un siglo de publicaciones científicas sobre la temática, son el índice de 'disimilitud' propuesto por Duncan y Duncan (1955) y el índice de 'exposición' 
propuesto por Bell (1954). El primero apunta a detectar la uniformidad y el segundo, la interacción.

El índice de disimilitud se basa en comparar la distribución del grupo minoritario con respecto del mayoritario y se define como:

$$
D=\frac{1}{2} \sum_{i=1}^{n}\left|\frac{x_{i}}{X}-\frac{y_{i}}{Y}\right|
$$

donde $x_{\mathrm{i}}$ es la población del grupo minoritario en la unidad espacial i, $X$ la población total del grupo minoritario en la ciudad, $y_{\mathrm{i}}$ es el número de individuos del grupo mayoritario en cada sección censal, e $Y$ el total de población de este grupo en la ciudad.

El índice de disimilitud está acotado entre cero (mínima segregación) y uno (máxima segregación), y también admite una interpretación en forma de porcentaje del grupo analizado que debe cambiar de unidad para alcanzar la igualdad en la distribución.

Por su parte, el índice de exposición sirve para conocer el grado de contacto potencial o la posibilidad de interacción entre miembros de dos grupos diferentes. A diferencia de los indicadores de uniformidad, los de exposición tienen en cuenta la representatividad de los grupos en la población total. Una distribución similar de dos grupos en el espacio urbano que puede dar lugar a índices de uniformidad nulos, no tiene por qué reflejar una situación de fuerte interacción entre sus miembros.

El índice de exposición se define de la siguiente forma (Bell, 1954; White, 1986):

$$
{ }_{x} P_{y}=\sum_{i=1}^{n}\left[x_{i} / X\right]\left[y_{i} / t_{i}\right]
$$

donde $x_{i}$ es la población del grupo $X$ en la unidad espacial $i, y_{i}$ es la población del grupo $Y$ en la unidad espacial $i, t_{i}$ es la población total en la unidad espacial $i, X$ es la población del grupo $X$ en la ciudad, y $n$ es el número de unidades espaciales en la ciudad.

El índice de interacción (Bell, 1954), está acotado entre cero y uno, pero tiene una interpretación opuesta al anterior. Por ejemplo, si este índice toma el valor 0,2 , se interpreta que de media, en una unidad donde reside un miembro del grupo $X$, dos individuos de cada diez son del grupo $Y$ (si solo hay dos grupos de población). En consecuencia, en las situaciones más segregativas tomará valores pequeños.

Algunos autores (Stearns y Logan, 1986) dan más importancia a este índice que a las medidas de uniformidad; según ellos, medir la probabilidad de interacción es clave, ya que la misma fomenta la integración en el idioma del país y puede propiciar, 
por ejemplo, matrimonios mixtos y, en general, todo proceso de asimilación. Por el contrario, otros autores (Bertrand y Chevalier, 1998) argumentan que las relaciones sociales no se limitan al espacio residencial, sino que también están presentes en otros ámbitos como el trabajo, las relaciones de amistad y otras; por lo tanto, no podemos acotar estrictamente al lugar de residencia la interacción entre los grupos.

La asunción general en los índices clásicos era que en caso de no segregación cada unidad censal mostraría el mismo porcentaje de una minoría que en la ciudad en su conjunto; por el contrario, una segregación completa mostraría unidades censales compuestas completamente por un grupo minoritario y el resto de las unidades no contendría población de este grupo.

Esta suposición es errónea. En teoría es posible que una ciudad tenga un patrón de segregación completa y al mismo tiempo lograr un valor de cero en los índices de segregación mencionados anteriormente.

Para ilustrar este punto, en forma exageradamente simple, supongamos una ciudad de cuatro unidades censales que son iguales en su extensión y en su población. En el primer caso (a) se asume que los límites raciales, étnicos y/o socioeconómicos coinciden con los límites de la unidad censal y que toda la población minoritaria, la segregación de los cuales se va a medir, se encuentra en una unidad tal como se representa en la Figura $\mathrm{N}^{\circ} 2$ (a). Con este patrón de distribución, cualquier índice de los expuestos funcionaría perfectamente y reflejaría la correcta intensidad de la segregación con un valor de uno para la uniformidad o cero para la exposición. Ahora, vamos a considerar otra ciudad (b), en la cual la minoría analizada no escogió su lugar de residencia sobre la base de los límites censales y, aunque se encuentre completamente segregada como en (a), la minoría considerada se encuentra localizada alrededor de la intersección de los límites de las cuatro unidades censales, superpuesta a todos ellos como se muestra en la Figura $\mathrm{N}^{\circ} 2$ (b).

Si la población minoritaria se distribuye uniformemente en los cuatro cuadrantes y cae en proporciones iguales en las cuatro unidades censales, los índices expuestos darían una puntuación de cero, indicando que no hay segregación. La cuestión es que estaríamos llegando a conclusiones equivocadas, ya que la segregación es igual de intensa en el segundo caso como en el primero. La única diferencia en los dos casos es que los límites, raciales, étnicos y/o socioeconómicos, coinciden con los límites censales en el primer caso y no en el segundo. Es justamente la diferencia en la ubicación geográfica de la minoría en relación a los límites de las unidades censales lo que determina la diferencia entre una puntuación de cero y una puntuación de uno. ${ }^{2}$

El problema de las unidades espaciales en las medidas de segregación y otras operaciones de análisis espacial no se agota en estas líneas; para profundizar sobre esta cuestión recomendamos la lectura de Openshaw 
Figura $\mathrm{N}^{\circ}$ 2. Error inducido por la delimitación de las unidades censales
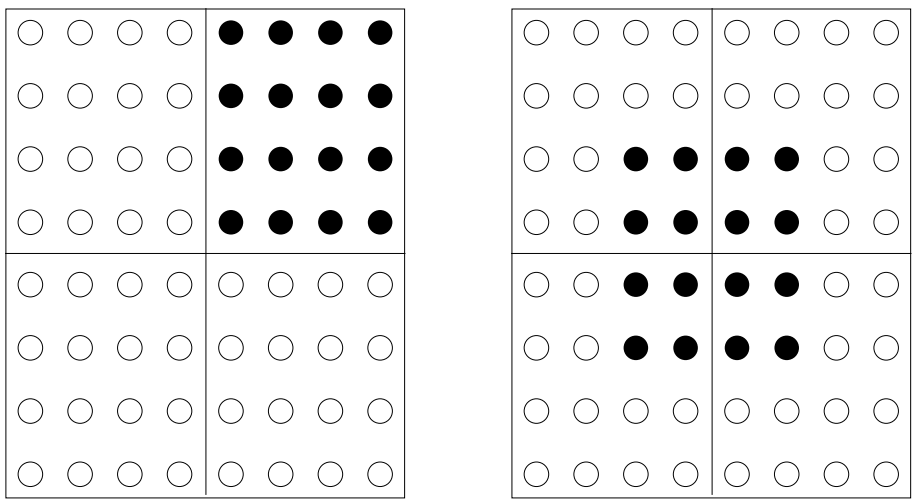

(a) (b)

Fuente: elaboración propia en base a Cowgill y Cowgill (1951).

En el mejor de los casos, si todas las secciones censales de todas las ciudades tuvieran extensiones de igual tamańo e iguales criterios socioeconómicos para su delimitación, los índices podrían dar lugar a algunas comparaciones significativas. Pero, en la realidad, los límites censales no son uniformes ni dentro de una misma ciudad, ni menos aún entre diferentes ciudades.

Así planteada la situación, cualquier índice que se base solamente en unidades censales como unidad de medición generará resultados poco confiables, siendo afectados por lo que en los antecedentes metodológicos se conoce como "el problema del tablero de ajedrez" y/o "el problema de la unidad espacial modificable" (White, 1983, p. 1010; Openshaw, 1984, p. 4; Reardon y O’Sullivan, 2004, p. 123).

Más allá de estas consideraciones estrictamente espaciales, otras objeciones matemáticas sobre las propiedades de los índices tradicionales basados en unidades censales fueron claramente explicitadas. Sobre el índice de disimilitud se ha señalado que no cumple con el criterio de transferencia, ${ }^{3}$ que indica cómo la segregación debe cambiar en respuesta al movimiento de personas en el espacio urbano. También se critica la hipotética igualdad como categoría opuesta a la segregación, sugiriendo que sería más apropiado considerar una situación de

(1984), disponible en: http://qmrg.org.uk/files/2008/11/38-maup-openshaw.pdf [abril 2013].

3 Transferencia: se refiere a que si un individuo del grupo $m$ es trasladado desde la subunidad $i$ a la subunidad $j$, en donde la proporción de personas del grupo $m$ es mayor en la subunidad $i$ que en $j$, entonces la segregación se reduce. 
aleatoriedad. Se demostró a su vez cómo estos índices se ven afectados por diferencias en la proporción de las minorías en la población, incumpliendo el criterio de invariación de composición, ${ }^{4}$ imposibilitando así los estudios comparativos entre ciudades, de la misma manera que se ven afectados por el tamańo (número de viviendas) de la unidad espacial de análisis, incumpliendo con el criterio de invariación de tamaño (Cortese, Falk y Cohen, 1976; Winship, 1977; James y Tauber, 1985; White, 1986).

Sin duda alguna, hay más consenso sobre el empleo de índices de interacción sobre el índice de disimilitud; sin embargo, también se señala que es afectado por el tamaño relativo de los grupos (Massey y Denton, 1988; Iceland y Nelson, 2008).

A partir de estas observaciones se publicaron artículos críticos que sugerían perfeccionar los índices tradicionales para la medición de la segregación; algunos intentaron resolver los problemas derivados de las unidades espaciales de análisis sobre las que las fórmulas se aplican, y otros proponían correcciones que dieran la posibilidad de incorporar múltiples categorías a las mediciones. Ambas cuestiones pretenden ser recuperadas en los apartados siguientes.

\section{La inclusión de la espacialidad en las medidas de segregación}

Varios índices han sido propuestos para incorporar la espacialidad en las medidas de segregación; la gran mayoría de ellos son modificaciones del índice de disimilitud, al cual le incorporan alguna ponderación a las relaciones de contigüidad espacial entre las unidades censales o calculan la distancia entre ellas con el fin de captar los 'esfuerzos de relocalización' necesarios para lograr la uniformidad. Dentro de este grupo se pueden destacar el 'índice de disimilitud corregido con una matriz de contigüidad binaria' de Morril (1991); el 'índice de disimilitud corregido por la longitud de la frontera' de Wong (1993) y el 'índice de disimilitud basado en la distancia’ de Jakubs (1981). Sin embargo, como cada una de estas medidas son una generalización del índice de disimilitud, no cumplirán necesariamente con ninguno de los criterios que este no cumple, como son los de transferencia y el de invariación de composición (James y Tauber, 1985).

4 Invariación de composición: si el número de personas del grupo $m$ en cada subunidad se multiplica por una constante $p$ y el número y distribución de personas de los grupos restantes permanecen sin cambios, entonces la segregación no varía.

5 Invariación de tamańo: si el número de personas de cada grupo $m$ en cada subunidad $j$ es multiplicado por una constante $p$, la segregación no varía. 
Dos propuestas consideramos que deben ser destacadas en este apartado. Una asociada a la medición de la uniformidad como es el 'índice de proximidad espacial' de White (1983), y otra orientada a la medición de la interacción: el 'índice de exposición decreciente con la distancia’ de Morgan (1983).

El primero fue desarrollado por White (1983) y propone una metodología que combina la lógica del índice de disimilitud con la inclusión de la distancia física entre las unidades censales. El resultado es un algoritmo que opera sobre la base del grupo mayoritario y minoritario, solo que incluye la distancia física entre ellos, lo que se hace usando dos supuestos:

a) que la distancia promedio entre las personas de cada subdivisión censal es igual a $0.6 \sqrt{A}$ (donde $A$ es la superficie de cada unidad censal) y;

b) que los residentes de cada subdivisión censal se localizan en el centroide, por lo tanto asume que la distancia de las personas entre unidades censales es igual a la distancia existente entre estos centroides.

Para medir cómo la interacción social cambia con la distancia, White (1983) emplea cuatro funciones alternativas, recomendando como la mejor opción donde $d_{i j}$ es la distancia lineal entre los centroides de los segmentos $i$ y $j$, mientras que el exponente negativo del coeficiente supone que la probabilidad de relaciones intragrupos decrece rápidamente con la distancia, tal como se representa en la Figura $\mathrm{N}^{\circ} 3$.

Figura $\mathrm{N}^{\circ}$ 3. Función de decrecimiento con la distancia

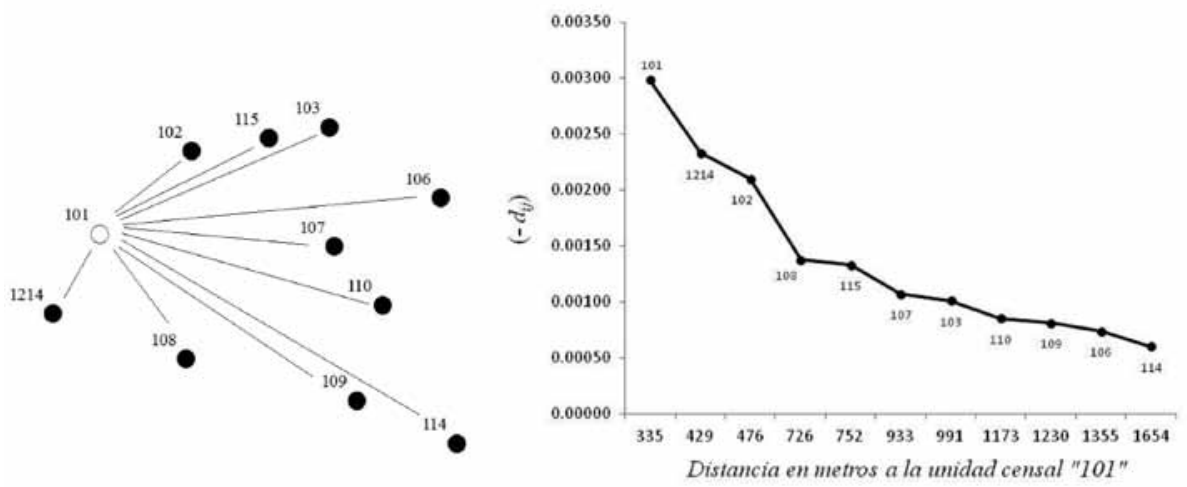

Fuente: elaboración propia.

A partir de estos supuestos es posible calcular: a) la distancia media de las personas en la unidad territorial superior sin relación con la pertenencia a grupos; b) 
la distancia media entre individuos del grupo mayoritario; c) la distancia media entre individuos del grupo minoritario, y d) la distancia media entre individuos del grupo minoritario e individuos del grupo mayoritario. Con estos parámetros, el autor propone un índice sintético que se interpreta como el promedio de las distancias intragrupo $\left(P_{11} / P_{00}\right.$ y $\left.P_{22} / P_{00}\right)$, ponderado por la fracción de cada grupo en la población. Más específicamente, la fórmula es:

$$
P=\frac{N_{1} P_{11}+N_{2} P_{22}}{\left(N_{1}+N_{2}\right) P_{00}}
$$

donde $N_{1}$ y $N_{2}$ son, respectivamente, el total de la población del grupo mayoritario y del grupo minoritario en la ciudad; $P_{00}$ es la distancia media de las personas en la unidad territorial superior sin relación con la pertenencia a grupos; $P_{11}$ es la distancia media entre individuos del grupo mayoritario y $P_{22}$ es la distancia media entre individuos del grupo minoritario. ${ }^{6}$

El valor de $P$ es igual a uno si no hay ninguna aglomeración socioespacial. Será mayor que uno cuando los miembros de un mismo grupo tienden a vivir juntos. Cuando $P$ es menor que uno indica una forma poco común de segregación, indicando que los miembros de un grupo tienden a vivir más cerca de los miembros del otro grupo.

Si bien el índice de proximidad espacial estaría superando los problemas del tablero de ajedrez y unidad espacial modificable, este no cumple varios de los criterios establecidos para evaluar las medidas espaciales de segregación (Reardon y O'Sullivan, 2004), como el criterio de interpretabilidad de la escala, ${ }^{7}$ ya que no posee un máximo teórico definido, imposibilitando de esta manera realizar estudios comparativos; ni tampoco cumple con los criterios de invariación de composición, transferencias e intercambios, descomposición espacial aditiva ${ }^{8}$ y descomposición aditiva de agrupamiento.?

La segunda medida es desarrollada por Morgan (1983), quien propone un 'índice de interacción ajustado por la distancia entre las unidades censales'. La limitación

Las formulas necesarias para obtener $P_{00}, P_{11}$ y $P_{22}$ se describen en White (1983, p. 1012).

7 Interpretabilidad de la escala: un índice de segregación debería ser igual a cero si cada entorno local tiene una composición igual a la de toda la población, y debería alcanzar su valor máximo (por lo general normalizado a uno) si el entorno local de cada individuo es monorracial, monoétnico o monoocupacional.

8 Descomposición aditiva espacial: si $X$ subáreas espaciales se agregan en $Y$ áreas espaciales más grandes, entonces una medida de segregación debe poder descomponerse en una suma de los componentes de la zona.

9 Descomposición aditiva por agrupamiento: si $M$ grupos se agrupan en $N$ supergrupos, entonces una medida de segregación debe poder descomponerse en una suma de los componentes dentro del supergrupo. 
que intenta superar el autor con su propuesta es que en realidad los miembros de una minoría no solo entran en contacto con individuos que se encuentran localizados dentro de su misma unidad censal (tal como lo considera el índice de exposición clásico), sino que lo hacen también con individuos localizados fuera de la unidad espacial de residencia. Al considerar un área más grande que la unidad censal, el contacto potencial aumenta considerablemente, aunque, al mismo tiempo, y a medida que uno se aleja de la unidad censal de origen, la probabilidad real de una minoría de interactuar con miembros del grupo mayoritario, disminuye.

El índice de exposición decreciente con la distancia $D P_{x y}$ propuesto por Morgan (1983) refleja estas dos fuerzas contrapuestas. La probabilidad de encontrar a un miembro de otro grupo disminuye en función de la distancia, mientras que el número de contactos potenciales aumenta:

$$
D P_{x y}=\sum_{i=1}^{n} \frac{x_{i}}{X} \sum_{j=1}^{n} \frac{k_{i j} y_{j}}{t_{j}} \quad \text { donde } \quad k_{i j}=\frac{t_{j}^{-d_{i j}}}{\sum_{i=1}^{n} t_{i j}^{-d_{i j}}}
$$

siendo $x_{i}$ el total de población del grupo $x$ en la unidad espacial $i ; y_{j}$ el total de población del grupo $y$ en la unidad espacial $j$; $t_{j}$ la población total de la unidad espacial $j ; t_{i j}$ la población total de las unidades espaciales $i$ y $j$, mientras que $d_{i j}$ es la distancia entre los centroides de las unidades espaciales $i$ y $j$.

Este índice puede ser interpretado como la probabilidad de que junto a una persona del grupo $X$ se encuentre una persona del grupo $Y$ en cualquier parte de la ciudad. Se obtendría un valor que oscila entre cero y uno, indicando en el primer caso una segregación máxima y en el segundo una integración completa.

\section{Medidas de segregación socioespacial multigrupo}

En un esfuerzo de síntesis, podemos decir que existen tres grandes atributos, no excluyentes entre sí, sobre los cuales la mayoría de los trabajos sobre segregación socioespacial se han basado para agrupar a los sujetos en categorías dentro de una ciudad. Uno de ellos, y sobre el cual nace este tipo de estudios, es el atributo de carácter racial, como suele aparecer en la mayoría de los estudios estadounidenses, centrados en la dicotomía negro-blanco, ${ }^{10}$ aunque hemos detectado que este 
tipo de abordajes no queda circunscrito a ese contexto regional, sino que también existen abordajes de segregación racial en numerosos países europeos, africanos, asiáticos y sudamericanos.

El segundo de los atributos tradicionalmente empleados es de carácter étnico. Aquí, las características culturales, como hábitos y costumbres, expectativas de vida y creencias, lengua y religión del país de origen, entre otras, serán determinantes para el agrupamiento de los sujetos en áreas residenciales. Al realizar este tipo de categorización, las medidas clásicas que emplean grupos dicotómicos ya no resultan apropiadas, a excepción de que se aborden de a pares, como suele aparecer en muchos artículos. Los antecedentes sobre segregación socioespacial étnica abundan inicialmente en las universidades europeas por la impronta multicultural y dinámica migratoria que caracteriza al continente, si bien en el contexto de la globalización -como fase actual de evolución del capitalismo- esta categorización se vuelve ineludible en todas las grandes ciudades del mundo.

El tercer atributo es de carácter socioeconómico, mayoritariamente representado por la calificación y/o categoría ocupacional de los sujetos, el nivel de ingresos, la rama de actividad económica que desempeńan, combinados en muchas ocasiones con otros atributos como el sexo, la edad y el nivel educativo, entre los más frecuentes. Esta es la forma de agrupamiento más usual en los estudios de segregación socioespacial en la actualidad y es la que mayoritariamente se emplea en el contexto urbano latinoamericano. Ello, básicamente porque el nivel de ingresos es el factor que determina las capacidades económicas reales de las familias para afrontar los costos de acceder a determinadas áreas residenciales en una ciudad, ya que los terrenos más aptos serán progresivamente utilizados por la población que pueda pagar por ellos, mientras que los terrenos con menores ventajas y peores localizaciones, por ende más baratos, serán utilizados para la construcción de viviendas de inferior calidad destinadas a la población de bajos recursos. En este proceso se van generando áreas de la ciudad que tienden a ser uniformes en términos de ingreso, de características culturales, valores y, sobre todo, en relación a la función que cumplen en la estructura social y división del trabajo.

En base a este espacio urbano en el cual la estructura social se vuelve más compleja para su análisis, es que Reardon y Firebaugh (2002) van a repensar y reconceptualizar las medidas de segregación orientadas ahora a enfoques que puedan abordar múltiples categorías sociales dentro de un colectivo urbano. Según los 
autores, estas medidas pueden ser definidas de cuatro maneras diferentes según lo que se interprete como segregación: a) como una función de la desproporcionalidad de representación de los grupos entre las unidades censales que conforman el área de estudio; b) como una disociación entre grupos y unidades censales; c) como una variación de la diversidad de las unidades censales, y d) como un promedio ponderado de los índices de segregación dicotómicos.

En búsqueda de alcanzar tal objetivo se ha propuesto una serie de medias de segregación multigrupo, dentro de las cuales se destaca el 'índice de la teoría de la información' de Theil (1972). A este se le han sumado otros esfuerzos menos difundidos como por ejemplo 'el índice de disimilitud multigrupo' (Sakoda, 1981) o 'el índice de exposición normalizado' (James, 1986).

El índice de la teoría de la información $H$ se puede definir como el promedio ponderado de las diferencias de representación proporcional entre los grupos en las subunidades censales y la ciudad en su conjunto (Theil, 1972). Este puede obtener valores entre cero y uno; dentro de este rango los valores bajos indican que los grupos al interior de las unidades censales poseen una representación similar a la de la ciudad en su conjunto, es decir, estarían expresando una máxima integración entre las categorías consideradas. En el extremo opuesto con valores altos cercanos a uno encontraríamos una situación en la que las unidades censales se componen de un solo grupo, es decir, máxima segregación. Este índice se formula de la siguiente manera:

$$
H=\sum_{i=1}^{n}\left[\frac{t_{i}\left(E-E_{i}\right)}{E T}\right] \text { donde } E=\sum_{m=1}^{M} \pi_{m} \ln \left(1 / \pi_{m}\right)
$$

siendo $\pi_{m}$ la proporción del grupo $m$ en la ciudad, $E$ y $E_{i}$ representan la diversidad (entropía) en la ciudad y en cada unidad censal, respectivamente; $T$ la población total; $t_{i}$ la población total en la unidad censal $i$, y $M$ el número de grupos.

Oportunamente, una serie de índices que pretenden avanzar en el mismo sentido fueron evaluados por Reardon y Firebaugh (2002) en base a una serie de criterios. Llegaron a la conclusión de que el más consistente (matemática y conceptualmente) entre los evaluados resulta ser el índice de la teoría de la información de Theil, siendo este el único (dentro de los seis índices analizados en su artículo) que cumple con el criterio de transferencia multigrupo y el criterio de descomposición multigrupo, al mismo tiempo que es uno de los dos que cumple con el criterio de descomposición organizacional. 
Más allá de que $H$ aparezca como el más destacado entre los índices multigrupo evaluados, una cuestión metodológicamente importante quedará por resolver: la de incorporar la espacialidad en las mediciones. Esto será resuelto finalmente por Reardon y O'Sullivan (2004) al desarrollar un conjunto de medidas espaciales multigrupo, empleando en sus cálculos superficies continuas de densidad, suavizadas mediante el método de interpolación picnofiláctico (Tobler, 1979), tanto para las dimensiones de uniformidad como de exposición.

Para medir la uniformidad, Reardon y O'Sullivan (2004) proponen el 'índice de la teoría de la información espacial' $(\widetilde{H})$ :

$$
\begin{gathered}
\widetilde{H}=1-\frac{1}{T E} \int_{p \in R} \tau_{p} \widetilde{E}_{p} d p \text { donde } E=-\sum_{m=1}^{M}\left(\pi_{m}\right) \log _{M}\left(\pi_{m}\right) \mathrm{y} \\
\widetilde{E}_{p}=-\sum_{m=1}^{M}\left(\tilde{\pi}_{m}\right) \log _{M}\left(\tilde{\pi}_{m}\right)
\end{gathered}
$$

siendo $T$ el total de la población, $E$ la entropía para toda la ciudad, $\tau_{\mathrm{p}}$ es la densidad de población en el punto $p, \widetilde{E}_{p}$ es la entropía para el entorno local de $p, M$ indica el número de grupos, $\widetilde{\pi}_{\mathrm{m}}$ la proporción del grupo $m$ en la ciudad, $\pi_{\mathrm{pm}}$ la proporción del grupo $m$ sobre el total de la población en el entorno local del punto $p$ y $\log _{\mathrm{M}}$ indica un logaritmo cuya base es $M$. Los autores utilizan un solo integral $\int_{q \in R}$ para denotar la suma sobre todos los puntos de una región.

Este índice es una medida de cuánto menos diversos son los entornos locales de los individuos, en promedio, con respecto a la diversidad que exhibe la ciudad en su conjunto $(R)$. Este índice, al igual que el análogo aespacial de Theil, es una medida que gravita en torno a los conceptos de entropía y redundancia. La entropía es una medida de desorden o incertidumbre derivada inicialmente de la termodinámica y luego adoptada por la teoría de la información. Sostiene que si la energía o la información (o para este caso particular las categorías sociales) se encuentran equitativamente distribuidas dentro de un sistema, la entropía es máxima (no hay desigualdades ni margen de acción para una redistribución). Si un sistema progresa hacia la igualdad, la entropía aumenta, y si se deteriora a expensas de un incremento de las desigualdades, la entropía disminuye y aumenta la redundancia. Es así que se define a los sistemas redundantes como aquellos caracterizados por una alta concentración y distribución desigual de la energía o información en su interior. 
A partir de estos conceptos es que el índice de la teoría de la información espacial $(\widetilde{H})$ será igual a uno cuando exista la máxima segregación socioespacial posible (máxima redundancia y mínima entropía), es decir, cuando cada entorno local a $p$ se encuentre habitado por un único grupo, como se observa en la Figura $\mathrm{N}^{\circ} 4$ (a). Por el contrario, si cada entorno local de $p$ tiene la misma composición por grupos que la ciudad en su conjunto, entonces la distribución espacial de cada grupo es uniforme y $\widetilde{H}$ será cero (mínima redundancia), indicando máxima integración socioespacial (máxima entropía) como se observa en la Figura $\mathrm{N}^{\circ} 4(\mathrm{~b})$.

Figura $\mathrm{N}^{\circ} 4$. Entropía y redundancia en el espacio urbano

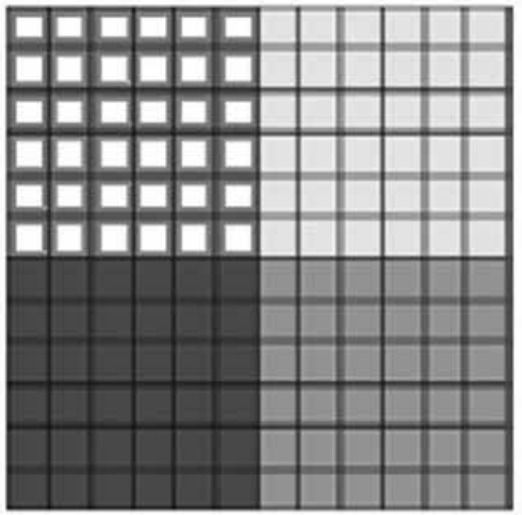

(a)

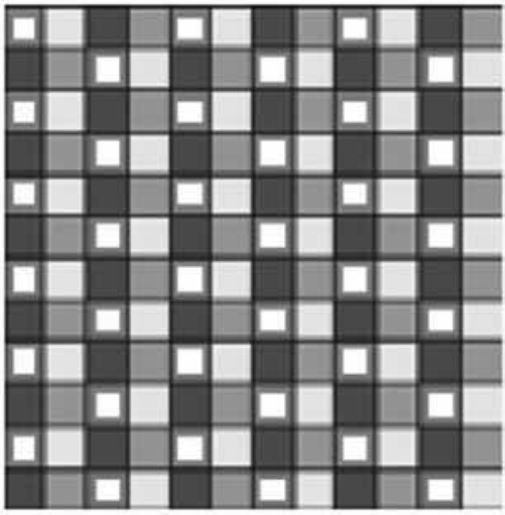

(b)

Fuente: elaboración propia.

Por otro lado, es posible aplicar la propiedad llamada 'equivalencia de entropía' para profundizar las interpretaciones sobre este indicador. Como muestra el Cuadro $\mathrm{N}^{\circ} 1$, a medida que aumenta el valor de $\widetilde{H}$ la distribución se hace más desigual y el potencial de redistribución se incrementa. A modo de ejemplo podemos decir que un valor de $\widetilde{H}=0,29$ indica que un $30 \%$ del grupo $m$ comparte el espacio con el $70 \%$ del grupo $n$, mientras que el $70 \%$ restante del grupo $m$ solo lo hace con el $30 \%$ del grupo $n$. En este caso existe un potencial de redistribución del $20 \%$ para alcanzar la máxima uniformidad. 
Cuadro $N^{\circ}$. Equivalencia en entropía y potencial de redistribución para eliminar la desigualdad

\begin{tabular}{|c|c|c|c|}
\hline Índice & \multicolumn{2}{|c|}{ Equivalencia en entropía } & $\begin{array}{l}\text { Potencial de } \\
\text { redistribución (\%) }\end{array}$ \\
\hline \multirow{2}{*}{ O } & 50 & 50 & \multirow{2}{*}{0} \\
\hline & 50 & 50 & \\
\hline \multirow{2}{*}{0,14} & 64 & 36 & \multirow{2}{*}{14} \\
\hline & 36 & 64 & \\
\hline \multirow{2}{*}{0,29} & 70 & 30 & \multirow{2}{*}{20} \\
\hline & 30 & 70 & \\
\hline \multirow{2}{*}{0,44} & 76 & 24 & \multirow{2}{*}{26} \\
\hline & 24 & 76 & \\
\hline \multirow{2}{*}{0,5} & 78 & 22 & \multirow{2}{*}{28} \\
\hline & 22 & 78 & \\
\hline \multirow{2}{*}{0,56} & 80 & 20 & \multirow{2}{*}{30} \\
\hline & 20 & 80 & \\
\hline \multirow{2}{*}{0,75} & 87 & 13 & \multirow{2}{*}{37} \\
\hline & 13 & 87 & \\
\hline
\end{tabular}

Fuente: elaboración propia, en base a Bacallao et al. (2002).

Para medir la exposición, Reardon y O'Sullivan (2004) proponen el 'índice de exposición espacial' $\left({ }_{m} \widetilde{P}_{n}^{*}\right)$, el cual se interpreta como el porcentaje promedio del grupo $n$ en los entornos locales de cada miembro del grupo $m$. El resultado estará comprendido entre cero, que indica ausencia de exposición al grupo $n, \mathrm{y}$ aumentará hasta llegar a uno, indicando que $m$ encontrará los máximos niveles de exposición posible al grupo $n$ :

$$
{ }_{m} \widetilde{P}_{n} *=\int_{q \in R} \frac{\tau_{q m}}{T_{m}} \widetilde{\pi}_{q n} d p
$$

donde $\tau_{q m}$ es la densidad de población del grupo $m$ en el punto $q, T_{m}$ es la proporción del grupo $m$ sobre el total de la población, $\widetilde{\pi}_{q n}$ es la proporción del grupo $n$ sobre el total de la población en el entorno local del punto $q$. Los autores utilizan un solo integral $\int_{\text {pep }}$ para denotar la suma sobre todos los puntos de una región.

Si bien la formulación original de ${ }_{m} \widetilde{P}_{n}^{*}$ no admite la incorporación de múltiples grupos, existen dos variantes en su ejecución mediante Sistemas de Información Geográfica que resultan muy interesantes a tal fin. Una es la posibilidad de obtener un valor de exposición para cada grupo versus la suma del resto de las categorías individuales; en este caso, $n$ estaría conformado por la sumatoria de los miembros de todos los grupos con excepción del grupo $m$. Otra opción es la posibilidad de organizar las categorías en forma ordinal y obtener valores de las sumas acumuladas versus el resto de la población. 


\section{Análisis empírico comparativo}

Se han escogido para los análisis empíricos 10 ciudades localizadas en la Provincia de Buenos Aires, las cuales se presentan en la Figura $\mathrm{N}^{\circ} 5$. Dos de ellas son aglomeraciones pequeñas comprendidas en el rango de 20.000 a 50.000 habitantes (Coronel Suárez y Trenque Lauquen); cuatro son aglomeraciones de tamaño intermedio incluidas en el rango de 50.000 a 100.000 habitantes (Azul, Luján, Olavarría y Pergamino); dos aglomeraciones incluidas en el rango de 100.000 a 250.000 habitantes (Tandil y San Nicolás de los Arroyos); una aglomeración en el rango de 250.000 a 500.000 (Bahía Blanca), y finalmente una aglomeración superior a los 500.000 habitantes (Mar del Plata). La segregación socioespacial estará significativamente influenciada por la densidad poblacional, existiendo una fuerte asociación entre el rango urbano y la tendencia centrífuga de localización residencial de los estratos de altos y medios ingresos, como resultado de una mayor penetración de pautas suburbanas de vida y acceso creciente al automóvil. En la Figura $\mathrm{N}^{\circ} 5$ pueden observarse tanto las diferencias relativas de superficie urbana como la tendencia de un patrón disperso en las ciudades más grandes, en contraposición a un ejido compacto para las ciudades más pequeñas.

También incidirá en la estructuración del espacio residencial la dinámica demográfica que ha caracterizado a cada una de ellas. En este sentido podríamos resaltar las ciudades que han tenido un crecimiento significativo como Trenque Lauquen y Luján, las cuales han mostrado una variación relativa durante el período 1991-2001 del 19\% y 16\%, respectivamente. En el otro extremo se encuentran las ciudades más grandes (Mar del Plata, Bahía Blanca y San Nicolás), las que muestran un crecimiento bajo que apenas supera el 5\%, indicando un síntoma de saturación de fuentes de trabajo e incapacidad de absorber la migración desde ámbitos rurales, pequeñas localidades y otras ciudades. Este grupo de personas migrantes son de nivel socioeconómico bajo en búsqueda de mejores condiciones de vida. Inicialmente arriban a las ciudades en búsqueda de áreas residenciales cercanas al centro, pero encuentran limitaciones a sus anhelos producto de los bajos ingresos percibidos por la falta de empleo, y el elevado valor del suelo y de las viviendas. Esto provoca un paulatino desplazamiento hacia la periferia, donde se accede a propiedades de bajo costo, alquileres baratos, conjuntos habitacionales de interés social o asentamientos ilegales, en la peor de las circunstancias, lo que termina profundizando la segregación socioespacial existente. El comportamiento del resto de las ciudades seleccionadas se asemeja al crecimiento promedio provincial en torno al $10 \%$.

Finalmente, y complementando el argumento anterior, será determinante la especialización y/o diversificación económica para obtener una mejor comprensión 
y contextualización de los índices de segregación en cada ciudad. La estructura económica, analizada según el producto bruto interno, demuestra que dentro de las ciudades seleccionadas algunas se encuentran sustentadas en una única actividad económica predominante, como ocurre en Trenque Lauquen, Coronel Suárez y Pergamino, que se orientan a la producción agrícola y ganadera; también Bahía Blanca, con un desarrollo intensivo en la industria manufacturera. Otro conjunto de ellas muestra una estructura económica basada en dos sectores de la economía; entre estas se encuentra Azul, que combina la producción agrícola y ganadera con la administración pública (Azul); San Nicolás, que combina la explotación de minas y canteras con la industria manufacturera (San Nicolás); Mar del Plata, que combina la industria manufacturera con actividades comerciales, de transporte y servicios inmobiliarios (Mar del Plata, con mayor peso en los servicios turísticos, y Luján y Olavarría en la industria manufacturera). Finalmente, Tandil presenta una economía diversificada entre los tres grandes sectores mencionados, distribuyendo equitativamente su producto bruto entre la producción agrícola y ganadera, la industria manufacturera y las actividades comerciales, de transporte y servicios inmobiliarios.

Figura $\mathrm{N}^{\circ} 5$. Localización de ciudades, rangos de población y ejidos urbanos

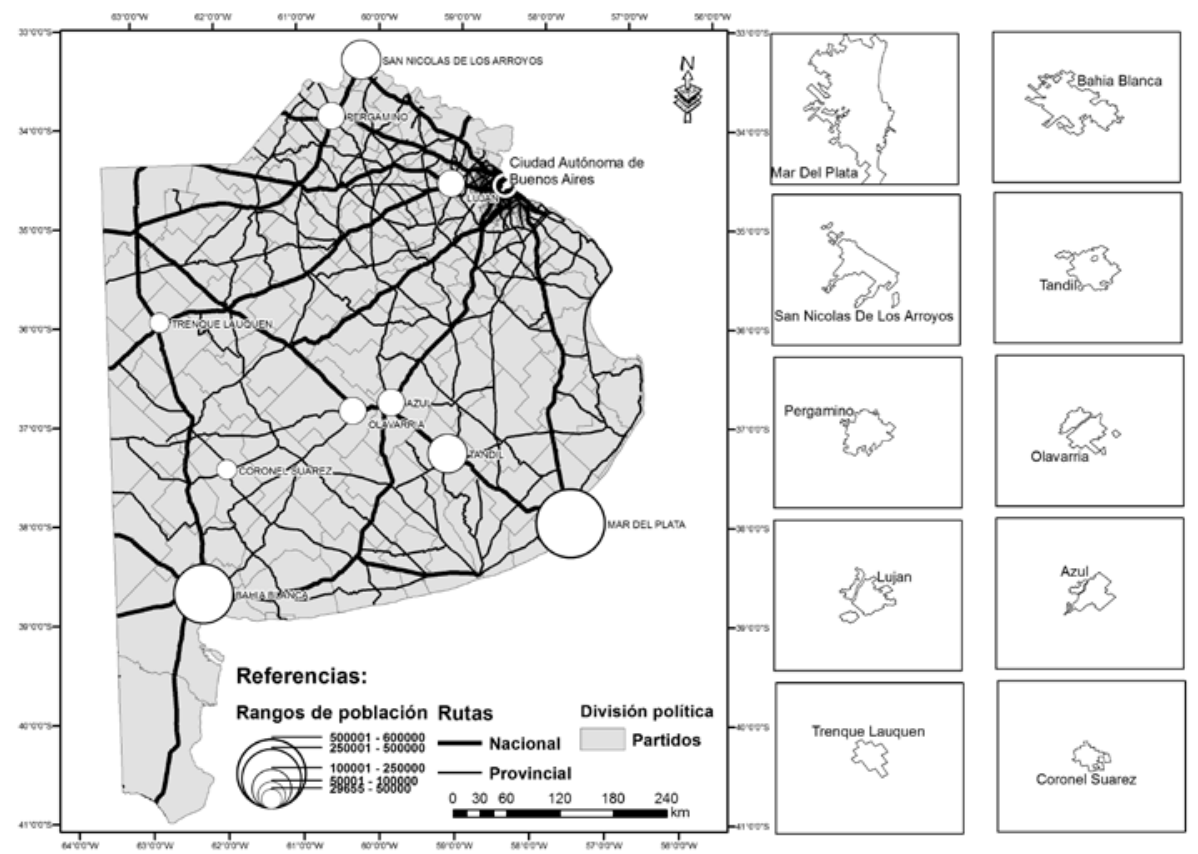

Fuente: elaboración propia, en base a datos suministrados por INDEC e IGN. 
Sobre estas ciudades se aplican seis índices de segregación socioespacial con el objetivo de medir la uniformidad e interacción, y comparar las variaciones que cada medida muestra sobre los mismos objetos empíricos de medición. Dos de los índices aplicados son los clásicos de disimilitud y exposición basados en unidades censales; otros dos son los índices espaciales de proximidad espacial y exposición decreciente con la distancia, y los últimos dos son los índices espaciales multigrupo de la teoría de la información y exposición espacial.

Se utilizará como atributo de clasificación y estratificación social la 'calificación de las ocupaciones' propuesta por el Instituto Nacional de Estadísticas y Censos en base al Clasificador Nacional de Ocupaciones (INDEC, 2001). Se trata de una variable objetiva del proceso de trabajo ${ }^{11}$ que mide la complejidad de las acciones desplegadas en dicho proceso. Es un atributo de las ocupaciones y no de las personas, que califica la complejidad de las tareas que indefectiblemente se encontrarán asociadas a una remuneración correspondiente. Las calificaciones se desagregan en:

Calificación profesional (CP): son aquellas en las que se realizan tareas múltiples, diversas y de secuencia cambiante, que suponen conocimientos teóricos de orden general y específico acerca de las propiedades y características de los objetos e instrumentos de trabajo, y de las leyes y reglas que rigen los procesos. Estas ocupaciones requieren de conocimientos adquiridos por capacitación formal específica y por experiencia laboral equivalente.

Calificación técnica (CT): son aquellas en las que se realizan tareas generalmente múltiples, diversas y de secuencia cambiante, que suponen paralelamente habilidades manipulativas y conocimientos teóricos de orden específico acerca de las propiedades y características de los objetos e instrumentos de trabajo, y de las reglas específicas que rigen los procesos involucrados. Estas ocupaciones requieren de conocimientos y habilidades específicas adquiridos por capacitación formal previa y/o experiencia laboral equivalente.

Calificación operativa (CO): son aquellas en las que se realizan tareas de cierta secuencia y variedad que suponen atención, rapidez y habilidades manipulativas, así como ciertos conocimientos específicos acerca de las propiedades de los ob-

11 En este sentido, "el trabajo es considerado como una actividad humana que a través de ciertas acciones e instrumentos efectúa una modificación planificada en su objeto, y cuya finalidad es la creación de un producto nuevo y diferente, de naturaleza tangible o intangible” (Crenzel et al., 2001, p. 3). 
jetos e instrumentos utilizados. Estas ocupaciones requieren de conocimientos y habilidades específicas adquiridas por capacitación previa y/o experiencia laboral.

No calificada (NC): son aquellas en las que se realizan tareas de escasa diversidad, utilizando objetos e instrumentos simples o, en muchos casos, el propio cuerpo del trabajador. Estas ocupaciones no requieren de habilidades o conocimientos previos para su ejercicio, salvo algunas breves instrucciones de inicio.

La información correspondiente a estas variables más aquella que refiere al total de habitantes de cada localidad, se encuentra originalmente agregada en los radios censales urbanos definidos por el INDEC para el censo 2001. A partir de esta fuente se elaboraron 10 matrices de datos originales, en donde las filas representan las unidades espaciales y las columnas las variables, y cuyas dimensiones se indican sintéticamente en el Cuadro $\mathrm{N}^{\circ} 2$.

Cuadro $\mathrm{N}^{\circ}$ 2. Dimensión de bases de datos originales para calificación de las ocupaciones y cantidad de habitantes según ciudades (2001)

\begin{tabular}{|c|c|c|c|c|c|c|c|c|c|c|}
\hline & $\begin{array}{c}\text { Mar del } \\
\text { Plata }\end{array}$ & $\begin{array}{c}\text { Bahía } \\
\text { Blanca }\end{array}$ & $\begin{array}{c}\text { San } \\
\text { Nicolás }\end{array}$ & Tandil & Pergamino & Olavarría & Luján & Azul & $\begin{array}{c}\text { Trenque } \\
\text { Lauquen }\end{array}$ & $\begin{array}{c}\text { Coronel } \\
\text { Suárez }\end{array}$ \\
\hline Filas (radios censales) & 762 & 284 & 131 & 107 & 92 & 95 & 84 & 58 & 34 & 25 \\
\hline Columnas (variables) & 5 & 5 & 5 & 5 & 5 & 5 & 5 & 5 & 5 & 5 \\
\hline
\end{tabular}

Fuente: elaboración propia, en base a datos suministrados por el INDEC (2001).

Los cálculos serán realizados mediante la implementación de dos aplicaciones informáticas, una totalmente libre, y otra que, si bien es libre, requiere de un Sistema de Información Geográfica licenciado como plataforma para su ejecución:

Geo-Segregation Analyzer: es un software de código abierto mediante el cual es posible calcular una cuarentena de índices de segregación residencial que la investigación sobre la temática nos ha dejado como legado. Tiene la particularidad de ser una aplicación multiplataforma (funciona con Windows, Mac, Linux y Unix), que permite leer el formato de archivos de ESRI Shapefiles y visualizarlos en una o más vistas (ver Figura $\mathrm{N}^{\circ}$ 6). Se puede descargar gratuitamente desde el sitio web del Spatial Analysis and Regional Economics Laboratory (SAREL) (Urbanisation, Culture Société, INRS), a través del siguiente enlace: http://geoseganalyzer.ucs. inrs.ca/ES/Inicio.html [julio 2013]. 
Figura $N^{\circ}$ 6. Geo-Segregation Analyzer

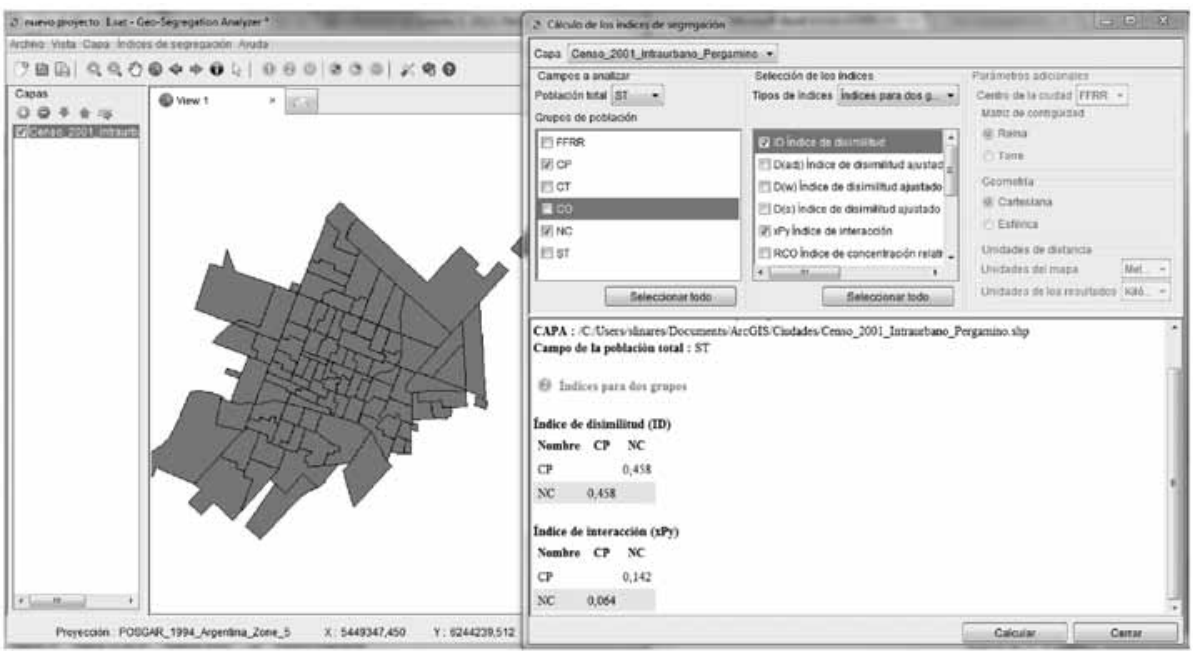

Fuente: elaboración propia.

SpatialSeg: es una aplicación programada para ejecutar sobre el Sistema de Información Geográfica ArcGIS 9.2 o 9.3 (ver Figura N 7), empleando Visual Basic para Aplicaciones (VBA). Tiene la particularidad de obtener unos cinco índices espaciales de segregación (y sus análogos aespaciales), mediante el empleo de superficies continuas de densidad que se obtienen de aplicar el método de interpolación picnofiláctico suavizado. Puede descargarse gratuitamente desde el sitio web del Population Research Institute (Pennsylvania State University) a través del siguiente enlace: http://www.pop.psu.edu/services/GIA/research-projects/mss [julio 2013]. 
Figura $N^{\circ}$ 7. SpatialSeg

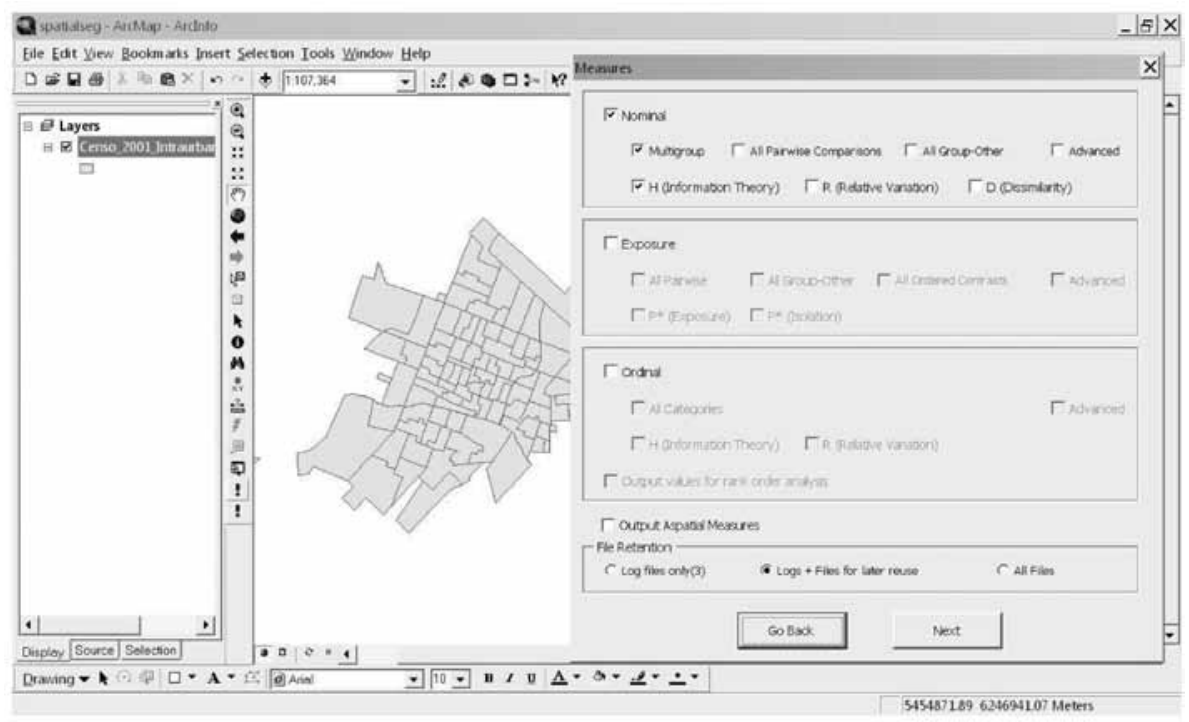

Fuente: elaboración propia.

Los resultados se presentan en el Cuadro $\mathrm{N}^{\circ} 3$ y en el Cuadro $\mathrm{N}^{\circ} 4$. En el primero se muestra el valor obtenido para cada medida seleccionada, el ranking de segregación de las ciudades, ordenado de tal forma que las primeras muestren la peor situación relativa y las últimas, los patrones de máxima integración. En el segundo se muestra la correlación existente entre los índices considerados, y entre los índices y la cantidad de habitantes.

Cuadro $\mathrm{N}^{\circ}$ 3. Comparación de medidas de segregación socioespacial para 10 ciudades medias bonaerenses (2001)

\begin{tabular}{|c|c|c|c|c|c|c|c|c|c|c|c|c|c|}
\hline \multirow[t]{2}{*}{ Ciudades } & \multirow{2}{*}{$\begin{array}{l}\text { Cantidad } \\
\text { de } \\
\text { habitantes }\end{array}$} & \multicolumn{4}{|c|}{$\begin{array}{c}\text { Medidas dicotómicas* } \\
\text { aespaciales }\end{array}$} & \multicolumn{4}{|c|}{$\begin{array}{c}\text { Medidas dicotómicas } \\
\text { espaciales }\end{array}$} & \multicolumn{4}{|c|}{$\begin{array}{c}\text { Medidas multignupo }{ }^{* *} \\
\text { espaciales }\end{array}$} \\
\hline & & $D$ & Remilas & ${ }_{x} P_{y}$ & Rembers & $P$ & Remeng & $D P_{y y}$ & Roultang & $\hat{H}$ & Remiby & $\vec{P}_{n}^{*}$ & 2 matas \\
\hline Mar del Plata & 541733 & 0.552 & 2 & 0.064 & 7 & 1.194 & 1 & 0.076 & 3 & 0.070 & 2 & 0.760 & 5 \\
\hline Bahía Blanca & 274509 & 0.537 & 3 & 0.068 & 9 & 1.163 & 2 & 0.080 & 8 & 0.064 & 3 & 0.759 & 4 \\
\hline San Nicolás & 125408 & 0.608 & 1 & 0.049 & 1 & 1.153 & 3 & 0.071 & 2 & 0.081 & 1 & 0.742 & 1 \\
\hline Tandil & 101010 & 0.472 & 7 & 0.070 & 10 & 1.089 & 4 & 0.082 & 9 & 0.053 & 4 & 0.779 & 8 \\
\hline Pergamino & 85487 & 0.457 & 8 & 0.064 & 8 & 1.046 & 7 & 0.088 & 10 & 0.051 & 6 & 0.780 & 9 \\
\hline Olavarria & 83738 & 0.490 & 6 & 0.059 & 5 & 1.061 & 5 & 0.077 & 7 & 0.051 & 5 & 0.769 & 6 \\
\hline Luján & 67266 & 0.454 & 9 & 0.061 & 6 & 1.057 & 6 & 0.076 & 5 & 0.046 & 8 & 0.776 & 7 \\
\hline Azul & 53054 & 0.524 & 5 & 0.058 & 4 & 1.044 & 8 & 0.077 & 6 & 0.046 & 9 & 0.751 & 3 \\
\hline Trenque Lauquen & 30764 & 0.530 & 4 & 0.053 & 3 & 1.029 & 9 & 0.076 & 4 & 0.046 & 7 & 0.743 & 2 \\
\hline Coronel Suárez & 22624 & 0.407 & 10 & 0.052 & 2 & 1.020 & 10 & 0.061 & 1 & 0.038 & 10 & 0.793 & 10 \\
\hline & & 0.50 & & 0.06 & & 1.09 & & 0.08 & & 0.05 & & 0.77 & \\
\hline
\end{tabular}

- No calificados (NC) y Calificación profesional (CP)

** No calificados (NC), Calificación profesional (CP), Calificación Técuica (CT) y Calificación Operativa (CO)

Fuente: elaboración propia. 
Cuadro $\mathrm{N}^{\circ} 4$. Matriz de correlaciones entre medidas de segregación socioespacial y cantidad de habitantes

\begin{tabular}{cccccccc}
\hline & $\begin{array}{c}\text { Cantidad } \\
\text { de } \\
\text { habitantes }\end{array}$ & $\begin{array}{c}\text { Medidas } \\
\text { dicotómicas } \\
\text { aespaciales }\end{array}$ & \multicolumn{2}{c}{$\begin{array}{c}\text { Medidas } \\
\text { dicotómicas } \\
\text { espaciales }\end{array}$} & $\begin{array}{c}\text { Medidas } \\
\text { multigrupo } \\
\text { espaciales }\end{array}$ \\
\cline { 3 - 9 } & & $D$ & ${ }_{x} P_{y}$ & $P$ & $D P_{x y}$ & $\widetilde{H}$ & $\widetilde{P}_{n}^{*}$ \\
\hline$D$ & 0.45 & 1 & - & - & - & - & - \\
${ }_{x} P_{y}$ & 0.41 & -0.19 & 1 & - & - & - & - \\
$P$ & 0.86 & 0.69 & - & 1 & - & - & - \\
$D P$ & 0.12 & 0.08 & 0.71 & 0.10 & 1 & - & - \\
$\tilde{H}_{x y}$ & 0.62 & 0.82 & - & 0.89 & - & 1 & - \\
$\tilde{P}_{n}^{*}$ & -0.19 & - & 0.33 & - & -0.07 & -0.53 & 1 \\
\hline
\end{tabular}

Fuente: elaboración propia.

Analizaremos en primera instancia los resultados globales que se muestran en el Cuadro $\mathrm{N}^{\circ} 3$, según las dos dimensiones consideradas: uniformidad e interacción. Con respecto a la uniformidad y basándonos en $D$ (es decir, sin tener en cuenta las relaciones topológicas entre las unidades censales y considerando solo dos grupos de población en la ciudad, como CP y NC), en promedio deberíamos relocalizar a aproximadamente un $50 \%$ de los miembros de uno de los grupos para obtener un patrón uniforme, es decir, los miembros de las categorías socioocupacionales antagónicas presentan un alarmante patrón de distribución diferenciado entre ellos y aglomerados entre sí. El valor promedio resulta ser significativamente alto, y más aún si se considera que existen casos en los que la segregación llega al umbral crítico del 60\%, como ocurre con San Nicolás, o cercano a él, como ocurre con el 55\% en Mar del Plata.

Similar comportamiento muestra el índice $P$, alejándose significativamente de uno en las ciudades de Mar del Plata, Bahía Blanca y San Nicolás, lo cual indica que en ellas los miembros de un mismo grupo tienden a vivir juntos. En el otro extremo se encuentran las ciudades de Trenque Lauquen y Coronel Suárez, donde los valores cercanos a uno indican que no habría tendencia a la aglomeración socioespacial. ${ }^{12}$

Siguiendo la misma tendencia, aunque con una situación mucho menos alarmante, observamos los resultados de $\widetilde{H}$ como producto de relativizar el análisis al

12 Queda en evidencia el problema de interpretabilidad de la escala al no contar con un máximo y mínimo predeterminado, lo que hace difícil analizar este índice en análisis comparativos. 
total de las categorías ocupacionales (y no solo entre $\mathrm{CP}$ y NC), donde el potencial de redistribución para lograr la igualdad en promedio es solo del 5\%, y donde los casos extremos, San Nicolás y Mar del Plata, no superan el $8 \%$.

La variación que existe entre $D$ y $\widetilde{H}$ en principio se debe al sobredimensionamiento de las diferencias entre categorías minoritarias en el primer caso y a la dilución de esas diferencias al incorporar a la población total en el segundo. Es decir, mientras que los grupos CP y NC solo representan 28\% del total de la Población Económicamente Activa, las dos categorías restantes, CT y CO, son las más numerosas y representan el $72 \%$. Esto implica que, al analizar la diversidad multigrupo en los entornos locales, un aumento o disminución de representación de los individuos pertenecientes a $\mathrm{CP}$ o $\mathrm{NC}$ en un punto determinado, posea un menor impacto en el índice que si los cambios se dieran en las categorías más populosas como son CT y CO. A su vez, ha sido posible corroborar en trabajos anteriores que CT y CO presentan un patrón de distribución uniforme, mostrando un bajo potencial de distribución que no supera el 3\%. Esto complementa lo expuesto anteriormente, ya que CT y CO no solamente inciden con mayor ponderación, sino que claramente sesgan los resultados en dirección a la uniformidad. En base a este argumento podríamos relativizar proporcionalmente el resultado de $D$ y decir que este solo representa un $28 \%$ de $\widetilde{H}$, y si sobre ese $28 \%$ la mitad se distribuye uniformemente, tendríamos que relocalizar solo a un 16,5\% de miembros pertenecientes a CP y NC. Podríamos seguir descontándole a $D$ las comprobadas sobreestimaciones provocadas por el incumplimiento de los principios de transferencia e invariancia de tamaño y la desconsideración de la espacialidad, y ajustar ese $16,5 \%$ hasta aproximarnos a $\widetilde{H}$. No obstante, es una cuestión que escapa a los objetivos de este artículo.

La dimensión de exposición admite una comparación entre los tres índices ya que se basan en la misma lógica. Es así que podemos decir en base $\mathrm{a}_{x} P_{y}$ que, en promedio, en cada unidad censal donde reside un miembro del grupo NC, uno de cada 20 son del grupo CP. Esta probabilidad de contacto potencial se amplía al contemplar toda la ciudad para realizar los cálculos en base a una función decreciente con la distancia para cada unidad censal $\left(D P_{x y}\right)$, en la cual por cada unidad donde reside un miembro del grupo NC, cerca de dos de cada 20 son del grupo CP. En términos generales esto significa que la interacción aumenta y la segregación disminuye al contemplar la ciudad en su conjunto en las mediciones. Aunque con poca diferencia entre las ciudades seleccionadas, se observan las peores situaciones relativas para ambos índices en las ciudades de Coronel Suárez y San Nicolás, mientras que Mar del Plata se encuentra en el tercer lugar. Al incorporar el resto de los grupos en las mediciones, las probabilidades de contacto de $\mathrm{NC}$ a las otras 
categorías aumentan indefectiblemente, mostrando que un 77\% de los miembros en los entornos locales de un miembro NC, pertenecen a algún otro grupo (CO, CT o CP). Las ciudades de Coronel Suárez y Mar del Plata vuelven a mostrar los valores más bajos de interacción entre las categorías; las ciudades de Azul y Bahía Blanca se incorporan a este grupo, mientras que el efecto de proporcionalidad entre categorías altera significativamente el orden entre el resto de las ciudades. $\mathrm{El}$ análisis de estos tres índices para esta dimensión permite concluir que, si bien la interacción entre $\mathrm{NC}$ y $\mathrm{CP}$ es muy baja, esto no implica que los miembros del grupo NC se encuentren aislados en los lugares donde residen, conclusión a la que podríamos haber erróneamente llegado si solo se hubieran contemplado grupos dicotómicos en las mediciones.

En segunda instancia, es posible verificar a partir del Cuadro $\mathrm{N}^{\circ} 3$ que la intensidad de la segregación socioespacial según ciudades presenta variaciones en los resultados según los índices de medición implementados y, según la dimensión de que se trate, menor es la variación en la uniformidad y más pronunciada en la exposición. Al comparar la medida clásica de uniformidad $(D)$ con sus análogas espaciales $(P)$ y multigrupo $(\widetilde{H})$, vemos descender la posición de las ciudades como máximo en cinco posiciones, como ocurre con la ciudad de Trenque Lauquen que se ubica en el cuarto lugar según $D$, y desciende al noveno según $P$ y séptimo según $\widetilde{H}$, a la cual se le podría sumar la ciudad de Azul, que se ubica en el quinto lugar según $D$ y desciende al octavo lugar según $P$ y noveno según $\widetilde{H}$. Las ciudades de Luján y Tandil también varían sus posiciones, aunque en tres puestos, con la diferencia de que ellas lo hacen ascendentemente, pasando del séptimo lugar según $D$ al cuarto según $\widetilde{H}$ en el caso de Tandil, y del noveno al sexto en el caso de Luján. Es decir, mientras que en las dos primeras ciudades se observa un sobredimensionamiento de la aglomeración socioocupacional, en las dos segundas se oculta este patrón por debilidades intrínsecas a las herramientas de medición.

Para la dimensión de exposición, la variación entre los índices es mucho más acusada, principalmente en los extremos como ocurre con Coronel Suárez y Bahía Blanca. En el primer caso, la interacción relativa aumenta al incluir la espacialidad y múltiples grupos, lo que se traduce en un descenso de nueve puestos en el ranking de segregación, desde el segundo lugar según el índice de exposición clásico al último empleando $\widetilde{H}$. En el otro extremo se encuentra Bahía Blanca, mostrando una reducción de cinco puestos en la interacción social al implementar $\widetilde{H}$.

Se deduce que contemplar la espacialidad y múltiples grupos en las mediciones produce un efecto directo en los resultados de los índices, lo cual debe ser tenido en cuenta al momento de realizar estudios empíricos comparativos. Mientras que la espacialidad debe ser ineludiblemente incorporada a todas las mediciones, la 
decisión de implementar índices multigrupo requiere una definición y justificación teórica más que metodológica, ya que dependerá en gran parte de las características de la estructuración de la sociedad en clases y de la categorización de grupos empleados para los análisis.

En tercera instancia, se corrobora la independencia entre las dimensiones de uniformidad y exposición, observando en el Cuadro $\mathrm{N}^{\circ} 4$ una insignificante correlación entre los pares $D \mathrm{y}_{x} P_{y}(r=-0.19)$ y entre $P$ y $D P_{x y}(r=0.10)$. Algo mayor es la asociación entre de $\widetilde{H} \mathrm{y}_{m} \widetilde{P}_{n}^{*}(r=-0.53)$, aunque no lo suficiente como para considerar redundante el aporte de cada medida por separado.

En cuarta instancia, con respecto a la incidencia del rango urbano en las medidas de segregación, se observa que con excepción de los índices tradicionales $D y_{x} P_{y}$, los que muestran un coeficiente de correlación similar, la incidencia va a tomar un comportamiento bien diferenciado según cada dimensión, siendo muy influyente para el caso de la uniformidad $(r \approx 0.7)$ e insignificante para la exposición $(r \approx 0.15)$. Los índices de uniformidad espaciales encuentran una correlación positiva y significativa como un efecto esperable al incorporar las relaciones topológicas de proximidad y adyacencia entre las unidades de medición, que permiten descubrir aglomeraciones socioespaciales en el espacio urbano. Es posible atender cómo las tres ciudades más grandes -Mar del Plata, Bahía Blanca y San Nicolás- muestran los valores más altos de aglomeración, mientras que las tres más pequeñas -Coronel Suárez, Trenque Lauquen y Azul- presentan una distribución más uniforme de las categorías sociales. El defecto de la desconsideración espacial de $D$ impide captar claramente esta correlación.

Para la exposición se observa una intrascendente influencia del rango urbano en los resultados de los índices ${ }_{x} P_{y}, D P_{x y} \mathrm{y}_{m} \widetilde{P}_{n}^{*}$, siendo mucho más importante y determinante el cambio provocado por la representatividad de los grupos incluidos en la medición. Es decir, los resultados obtenidos al aplicar ${ }_{x} P_{y}$ y $D P_{x y}$, donde solo se consideran dos categorías, son similares $(r=0.71)$, mientras que la incorporación del total de los grupos al aplicar ${ }_{m} \widetilde{P}_{n}^{*}$ muestra resultados muy diferentes con respecto a sus antecesoras $(r=0.33$ y -0.07$)$.

\section{Conclusión}

Las medidas implementadas en sí mismas son expresiones indirectas e imperfectas de una realidad demasiado compleja y demasiado multiforme como para poder ser completamente resumida por alguna ecuación matemática. La validez y correcta implementación e interpretación de su uso resultará de la combinación de su sofisticación metodológica, y del bagaje teórico y conceptual de que disponga el 
investigador para comprender la realidad y dar sustento a los resultados obtenidos de dichas aplicaciones.

Es fundamental conocer sobre los fundamentos teóricos y metodológicos que subyacen a las medidas de segregación, sobre todo si se desea realizar estudios comparativos. Ha sido empíricamente demostrado en este artículo cómo el solo hecho de cambiar la forma de medir la segregación altera los resultados entre las ciudades, pudiendo llevar a conclusiones equivocadas basadas en metodologías 'tradicionalmente' aceptadas.

Se corrobora la independencia entre las dimensiones de uniformidad y exposición; es posible observar una baja correlación entre los pares aespaciales, espaciales y multigrupo, lo cual ratifica el aporte singular de cada dimensión a la comprensión del proceso de segregación socioespacial.

La relación entre tamańo de las ciudades y uniformidad pareciera respetar cierta regularidad y esta resulta ser inversa. A partir de correlacionar la cantidad de habitantes con las medidas espaciales de uniformidad, se observa que las ciudades con mayor población muestran un patrón mucho más aglomerado que las pequeñas urbanizaciones, donde la uniformidad prevalece. Esto puede vincularse con el impacto que los procesos migratorios y de crecimiento poseen en aquellas localidades donde las fuentes de empleo y de espacio residencial encuentran un límite, aglomerando a población con escasos recursos en las periferias urbanas, áreas vulnerables o sin amenidades, y asignando a nuevas áreas exclusivas a los miembros de grupos de mayores ingresos mediante mecanismos de especulación inmobiliaria y procesos públicos-privados de fragmentación urbana que van mucho más allá de la función residencial, pues se incluyen las funciones comerciales, el ocio y el esparcimiento, el deporte y la educación. Mientras tanto, la exposición presenta un comportamiento independiente con respecto al rango urbano y, ciertamente, más influenciada por la representación proporcional de los grupos en cada ciudad. Es en este punto donde habrá que ahondar más aún, prestando mayor atención a los sectores de actividad económica que caracterizan a la oferta de empleo en cada ciudad, e intentar explicar aquellos comportamientos y potenciales grados de interacción en los ocupados en diferentes ramas económicas. De la misma manera, resultará indispensable para obtener una comprensión más completa contar en este punto con información del proceso histórico de segregación socioespacial y con información sobre la movilidad intraurbana de los habitantes.

Más allá de las falencias señaladas, es posible afirmar que en las ciudades medias de la Provincia de Buenos Aires la población según calificación de las ocupaciones muestra un patrón segregado. Dentro de las dimensiones prevalece la aglomeración más que el aislamiento; es decir, si bien se distribuyen espacialmente en forma 
aglomerada, esto no determina que las categorías se encuentren aisladas en las áreas residenciales. Este punto de partida estimula a avanzar en dos direcciones: por un lado, conocer acerca de la evolución de esta estructuración del espacio urbano, vale decir, si la segregación socioespacial manifiesta aumentos o retrocesos durante el transcurso de la última década; $y$, por otro, constatar algunos de los hallazgos obtenidos en este trabajo, como la fuerte relación existente entre uniformidad y rango urbano.

Finalmente, las aplicaciones Geo-Segregation Analyzer y SpatialSeg, que hicieron posible este análisis, se presentan como dos alternativas complementarias más que excluyentes, recuperando entre ambas la evolución de las discusiones metodológicas más importantes expuestas durante los últimos 50 años. A partir de su uso es posible aplicar prácticamente la totalidad de las medidas de segregación socioespacial existentes en la literatura, en una reducida fracción de tiempo y sin costo alguno, permitiendo de esta manera dedicarle más tiempo a la interpretación y comprensión de los resultados, que debe ser, a final de cuentas, el propósito fundamental de este tipo de aportes metodológicos.

Recibido febrero 28, 2013 Aprobado julio 9, 2013

\section{Referencias bibliográficas}

Apparicio, P., Fournier, E., Apparicio, D. (2012). Geo-Segregation Analyzer: a multi-platform application (version 1.0). Spatial Analysis and Regional Economics Laboratory (SAREL), INRS Urbanisation Culture Société. Montreal, Canadá.

Bacallao, J., Castillo Salgado, C., Schneider, M. C., Mujica, O. J., Loyola, E., Vidaurre, M. (2002). Índices para medir las desigualdades de salud de carácter social basados en la noción de entropía. Revista Panamericana de Salud Pública 12-6, 429-435. Doi: dx.doi. org/10.1590/S1020-49892002001200008.

Bell, W. A. (1954). Probability Model for the Measurement of Ecological Segregation. Social Forces 32, 357-364.

Bertrand, J. R., Chevalier, J. (1998). Logement et habitat dans les villes européennes. París: L'Harmattan, collection Géographie Sociale.

Burgess, E. W. (1925). The growth of city: an introduction to a research project. En Park, R. E., Burgess, E. W., Mckenzie, R. D. The City, 47-62. Chicago: The University of Chicago Press.

Castells, M. (1972). La cuestión urbana. Madrid: Siglo XXI.

Cortese, Ch., Falk, R. F., Cohen, J. (1976). Further considerations on the methodological analysis of segregation indices. American Sociological Review 41, 630-637. 
Cowgill, D. O., Cowgill, M. S. (1951). An index of segregation based on block statistics. American Sociological Review 16, 825-831.

Crenzel, E., Esses, M., Hoexter, P., La Rocca, C., Morales, N., Urso, M., Elizalde, M. L. (2001). Clasificador nacional de ocupaciones 2001. $5^{\circ}$ Congreso Nacional de Estudios del Trabajo. ASET, Facultad de Ciencias Económicas de la Universidad de Buenos Aires, Argentina. Doi: http://www.aset.org.ar/congresos/5/aset/PDF/URSOYOTROS.PDF.

Duncan, O., Duncan, B. (1955). A methodological analysis of segregation indexes. American Sociological Review 20, 210-217.

Grafmeyer, Y. (1994). Regards sociologiques sur la ségrégation. En Brun, J., Rhein, C. La ségrégation dans la ville. Concepts et mesures, 85-117. París: L’Harmattan.

Graham, S., O'Sullivan, D. (2007). A Brief Guide to Running the SpatialSeg Program in ArcGIS 9.2. Measuring Spatial Segregation Project. National Science Foundation. University of Pennsylvania, Pennsylvania, Estados Unidos. Doi: http://www.pop.psu.edu/ services/GIA/research-projects/mss.

Iceland, J., Nelson, K. (2008). Hispanic Segregation in Metropolitan America: Exploring the Multiple Forms of Spatial Assimilation. American Sociological Review 73, 741-765.

INDEC (2001). Censo Nacional de Población, Hogares y Vivienda 2001. Instituto Nacional de Estadísticas y Censos. Ministerio de Economía. Buenos Aires, Argentina.

Jakubs, J. (1981). A distance-based segregation index. Socio-Economic Planning Science. Elsevier 15 (3), 129-136.

James, F. (1986). A New Generalized “Exposure-Based” Segregation Index. Sociological Methods and Research 14, 301-316.

James, D. R., Tauber, K. E. (1985). Measures of segregation. Sociological Methodology 15, 1-32.

Linares, S. (2012). Dificultades metodológicas al medir la segregación: el problema del tablero de ajedrez y de la unidad espacial modificable. Geografía y Sistemas de Información Geográfica (GeoSIG) (GESIG-UNLU, Luján), Año 4, N 4, Sección II, 10-22. Doi: http://www. gesig-proeg.com.ar/geosig-1-2012.htm.

Martori, J., Hoberg, K. (2004). Indicadores cuantitativos de segregación residencial. El caso de la población inmigrante en Barcelona. Scripta Nova. Revista Electrónica de Geografía y Ciencias Sociales, Vol. VIII, No 169. Doi: http://www.ub.edu/geocrit/sn/sn-169.htm.

Martori, J., Hoberg, K., Madariaga, R. (2008). La incorporación del espacio en los métodos estadísticos: autocorrelación espacial y segregación. Actas del X Coloquio Internacional de Geocritica. Universidad de Barcelona, Barcelona. Doi: http://www.ub.edu/geocrit/xcol/195.htm.

Massey D. S., Denton N. A. (1988). The dimensions of residential segregation. Social Forces 67 (2), 281-315.

Morgan, B. A. (1983). Distance-Decay Interaction Index to Measure Residential Segregation. Area 15, 211-216. 
Morrill, R. (1991). On the measure of geographical segregation. Geography Research Forum $11,25-36$.

Musterd, S., Ostendorf, W. (1998). Urban Segregation and the Welfare State. Inequality and Exclusion in Western Cities. Londres: Routledge.

Openshaw, S. (1984). The modifiable areal unit oroblem. CATMOG 38. GeoBooks. Norwich, England. Doi: qmrg.org.uk/files/2008/11/38-maup-openshaw.pdf.

Reardon, S., Firebaugh, G. (2002). Measures of multi-group segregation. Sociological Methodo$\log y 32,33-67$.

Reardon, S., O’ Sullivan, D. (2004). Measures of spatial segregation. Sociological Methodology 34, 121-162.

Rodríguez, J. (2001). Segregación residencial socioeconómica: ¿qué es?, ¿cómo se mide?, ¿qué está pasando?, ¿importa? Serie Población y Desarrollo. CEPAL, ECLAC. Santiago, Chile. (2005). Midiendo la segregación residencial y sus determinantes demográficos con microdatos censales. Santiago: CELADE, CEPAL.

Sabatini, F. (2003). La segregación social del espacio en las ciudades de América Latina. Washington DC: División de Programas Sociales, Banco Interamericano de Desarrollo, Departamento de Desarrollo Sostenible.

Sabatini, F., Sierralta, C. (2006). Medición de la segregación residencial: meandros teóricos y metodológicos, y especificidad latinoamericana. Documento de Trabajo No 38. Instituto de Estudios Urbanos y Territoriales, Pontificia Universidad Católica de Chile, Santiago, Chile.

Sakoda, J. N. (1981). A generalized index of dissimilarity. Demography 18 (2), 245-250.

Schteingart, M. (2001). La división social del espacio en las ciudades. Perfiles Latinoamericanos $9,13-31$.

Sposito, M. E. B. (2005). A produção do espaço urbano em dez anos de GAsPERR: reflexão individual sobre uma trajetória coletiva (Sposito, E. S. Org). Produçao do espaço e redefiniçoes regionais: a construção de uma temática. Presidente Prudente: UNESP, FCT, GAsPERR.

Stearns, L. B., Logan, J. R. (1986). Measuring trends in segregation: three dimensions, three measures. Urban Affairs Quarterly 22, 124-150.

Theil, H. (1972). Statistical Decomposition Analysis. Amsterdam: North-Holland.

Tobler, W. (1979). Smooth Pycnophylactic Interpolation for Geographical Regions. Journal of the American Statistical Association, Vol. 74, N³67, 519-530.

White, M. (1983). The Measurement of Spatial Segregation. American Journal of Sociology 88, 1008-1018.

(1986). Segregation and Diversity Measures in Population Distribution. Population Index 52 (2), 198-221.

Winship, C. (1977). A re-evaluation of indexes of residential segregation. Social Forces 55 (4), 1058-1066.

Wong, D. (1993). Spatial Indices of Segregation. Urban Studies 30 (3), 559-572. 